\title{
Assessing anxiety-linked impairment in attentional control without eye-tracking: The
}

masked-target antisaccade task

Julian Basanovic ${ }^{1}$, Jemma Todd ${ }^{2}$, Bram van Bockstaele ${ }^{1,3}$, Lies Notebaert ${ }^{1}$, Frances Meeten ${ }^{4}$, Patrick J. F. Clarke ${ }^{5}$

${ }^{1}$ Centre for the Advancement of Research on Emotion, School of Psychological Science, The University of Western Australia

${ }^{2}$ School of Psychology, University of Sydney

${ }^{3}$ Research Institute of Child Development and Education, University of Amsterdam

${ }^{4}$ School of Psychology, University of Sussex

${ }^{5}$ Affective, Behavioural, and Cognitive Neuroscience Research Group, Curtin University

Corresponding author:

Julian Basanovic

julian.basanovic@uwa.edu.au

\section{Acknowledgements:}

The research reported in this manuscript is a product of the Cognition Emotion Research Collaboration Initiative (CERCI).

The research reported in this manuscript was supported in part by the Australian Research Council (Grant \#: FL170100167).

\section{Keywords:}

anxiety; attentional control; antisaccade 


\begin{abstract}
Contemporary cognitive theories of anxiety and attention processing propose that heightened levels of anxiety vulnerability are associated with a decreasing ability to inhibit the allocation of attention toward task-irrelevant information. Existing performance-based research has most often used eye-movement assessment variants of the antisaccade paradigm to demonstrate such effects. Critically however, eye-movement assessment methods are limited by expense, the need for expert training in administration, and limited mobility and scalability. These barriers have likely led to researchers using suboptimal methods of assessing the relationship between attentional control and anxiety vulnerability. The present study examined the capacity for a non-eye-movement based variant of the antisaccade task, the masked-target antisaccade task (Guitton et al., 1985), to detect anxietylinked differences in attentional control. Participants $(N=342)$ completed an assessment of anxiety vulnerability and performed the masked-target antisaccade task in an online assessment session. Greater levels of anxiety vulnerability predicted poorer performance on the task, consistent with findings observed from eye-movement methods and with cognitive theories of anxiety and attention processing. Result also revealed the task to have high internal reliability. Our findings indicate the masked-target antisaccade task provides a psychometrically reliable, low-cost, mobile, and scalable assessment of anxiety-linked differences in attentional control.
\end{abstract}




\section{Introduction}

Contemporary cognitive theories of anxiety and attention processing propose that heightened levels of anxiety vulnerability are associated with a decreased ability to inhibit the allocation of attention toward task-irrelevant information (Attentional Control Theory; Derakshan \& Eysenck, 2009; Eysenck et al., 2007). This proposal has been supported by recent meta-analytic findings demonstrating that participants with elevated anxiety vulnerability demonstrate reduced attentional control performance as compared to individuals lower in anxiety vulnerability (Shi et al., 2019). This phenomenon has been labelled an anxiety-linked impairment in attentional control.

A considerable body of literature examining this anxiety-linked impairment in attentional control has employed questionnaire measures to assess attentional control. In particular the Attention Control Scale developed by Derryberry and Reed (2002) has sought to measure attentional skills related to voluntary executive functions, using participants' responses on this scale as an index of attentional control ability. In their original study, the researchers observed poorer self-reported attentional control ability among participants with greater levels of anxiety vulnerability. One benefit of such self-report measures is that they demonstrate a high level of internal reliability (Fajkowska \& Derryberry, 2010; Ólafsson et al., 2011; Quigley et al., 2017) and are easily accessible and scalable in delivery (e.g. online delivery). Thus, the Attentional Control Scale is understandably favoured by researchers, and a number of investigators have employed the Attentional Control Scale to investigate attentional control amongst participants who vary in anxiety vulnerability (Bardeen \& Orcutt, 2011; Muris et al., 2004; Ólafsson et al., 2011; Schoorl et al., 2014). Critically however, the use of questionnaire measures to index cognitive processing is limited due to their measurement of one's beliefs about attentional control processes. 
Indeed, several recent studies have demonstrated no clear evidence of an association between participants' self-reported level of attentional control ability and their level of performance on attentional control assessment tasks (Quigley et al., 2017; Reinholdt-Dunne et al., 2009, 2013; Todd et al., 2020; Williams et al., 2017). Thus, evidence that scores on the scale reflect one's ability to control attentional processes is limited.

In contrast to questionnaire measures, researchers have also employed performancebased measures to assess attentional control. A performance-based assessment that has been frequently employed by researchers investigating anxiety-linked differences in attentional control is the antisaccade paradigm. The antisaccade paradigm (Hallett, 1978) involves a series of trials that each present an abrupt distractor stimulus in a left or right peripheral location onscreen. Participants are required to inhibit the reflexive orienting of attention towards the distractor stimulus when it appears and direct attention to the opposite screen position as rapidly as possible. More rapid movement of attention to the desired location, and fewer erroneous attention movements towards the distractor stimulus, are believed to represent better attentional control. When examining task performance amongst individuals who vary in anxiety vulnerability, investigators have observed that individuals with relatively heightened levels of anxiety vulnerability demonstrate poorer performance as compared to individuals with lower levels of anxiety vulnerability (Ansari \& Derakshan, 2010, 2011; Basanovic et al., 2018; Derakshan et al., 2009; Myles et al., 2020; Wright et al., 2014). Further research has also demonstrated modulation of anxiety-linked differences in performance on the antisaccade task via the adjustment of the content of the distractor stimulus (Chen et al., 2014; Reinholdt-Dunne et al., 2012; Wieser et al., 2009). Thus, the antisaccade paradigm has demonstrated sensitivity to anxiety-linked differences attentional control and has served as a useful tool in the 
investigation of anxiety-linked differences in the processing of neutral and emotional information.

At present, research focusing on the relationship between anxiety vulnerability and antisaccade performance has consistently assessed performance through the recording of eye-movements. The recording of eye-movements provides researchers with the benefit of measuring the accuracy and latency of participants' attentional movements across the duration of a trial. In addition, researchers have reported that eye-movement based measures hold a high level of internal reliability $\left(\alpha_{\text {cronbach }}=.85\right.$, Ettinger et al., $2003 ; r_{\text {spearman- }}$ Brown(SB) $=.95$, Myles et al., 2020). However, such approaches come with practical limitations including financial barriers, specialist operator training, and the inability to deploy assessments on a large scale.

Alongside assessments using eye-movement recording, researchers have developed approaches that instead utilise manual response latencies to index attentional control. For example, researchers have presented a visual target in the location opposite the distractor stimulus and recorded the speed at which participants are able to discriminate the identity of the target via a manual key-press (Ansari et al., 2008; Basanovic et al., 2017, 2020; Derakshan et al., 2009; Wright et al., 2014). Such tasks are readily accessible to researchers, require only minimal technical expertise to adopt, and can be easily complete on devices inlab and through the internet. However, while these approaches resolve the practical issues inherent to eye-movement assessments, estimates of the internal reliability of these methods have demonstrated modest reliability $\left(r_{\mathrm{SB}}=.59\right.$; Basanovic et al., 2020) and researchers have not detected anxiety-linked impairment in attentional control when using these tasks. 
Thus, the capacity for researchers to investigate the relationship between anxiety vulnerably and attentional control is presently impeded by the absence of accessible, scalable, and reliable performance-based assessment methods that have been demonstrated to be sensitive to anxiety-linked differences in attention control. One task that may help to solve this problem was developed by Guitton et al. (1985), labelled here the masked-target antisaccade assessment task. This task first presents a distractor stimulus followed by a visual target in an opposing screen location and participants are required to discriminate the identity of the target. Importantly, the target is presented only briefly (150 ms) before being masked. Thus, accurate discrimination of the target necessitates rapid attentional movement away from the initial distractor stimulus. The task indexes performance via target discrimination accuracy, with a greater number of accurate responses across trials reflecting greater attentional control. Notably, this task has been shown to be associated with other cognitive control processes theorised to be linked with attentional control (Friedman \& Miyake, 2004; Kane et al., 2001; Miyake et al., 2000; Roberts et al., 1994; Unsworth et al., 2011), and to hold a relatively high level of internal reliability ( $r_{S B}=.77$ to .87 ; Friedman \& Miyake, 2004; Miyake et al., 2000). Therefore, the masked-target antisaccade assessment approach stands as a promising performance-based method for investigating anxiety-linked differences in attentional control that circumvents the limitations of eye-movement and response-latency based approaches.

Critically, no research to date has sought to directly investigate whether the maskedtarget antisaccade assessment task demonstrates sensitivity to anxiety-linked differences in attentional control. Thus, the aim of the present study was to determine whether the masked-target antisaccade assessment approach is sensitive to anxiety-linked differences in attentional control. The study recruited individuals to complete a questionnaire measure of 
anxiety vulnerability followed by a masked-target antisaccade assessment task. Analyses examined the association between anxiety vulnerability and performance on the task and the internal reliability of the task. It was predicted that if the task is sensitive to anxietylinked differences in attentional control, then greater levels of anxiety vulnerability would be associated with poorer performance on the task.

\section{Method}

\section{Participants}

Recruitment of participants to this study was conducted online as part of the Cognition and Emotion Research Collaboration Initiative (CERCI) with researchers in Australia and the England. In total, 342 individuals ( 243 female, 96 male, 3 non-binary; Age $M=20.75$ years, $S D=4.79$ years) participated in the study. Invitations to participate were made available to students through undergraduate participant pools at Curtin University $(N=104)$, the University of Western Australia ( $N=77)$, and the University of Sydney $(\mathrm{N}=116)$, and via social media through the University of Sussex $(N=45)$.

\section{Materials}

\section{Depression, Anxiety, Stress Scales - Anxiety Scale}

The measure of anxiety was drawn from the 21-item version of the Depression, Anxiety, Stress Scales questionnaire (DASS-21; Lovibind \& Lovibond, 1995). The DASS-21 Anxiety Scale comprises seven self-report items that assess the degree to which participants experienced symptoms of anxiety during the previous week. Scores on the anxiety scale range from 0 to 21 with higher scores representing higher levels anxiety. The DASS-21 has been shown to have high test-retest reliability and high concurrent and construct validity among university student and general community populations (Antony et al., 1998). Internal consistency in the current study sample was excellent (Cronbach's alpha $=.87$ ). 


\section{Masked-target Antisaccade Assessment Task}

The Masked-target Antisaccade Assessment Task was delivered to measure attentional control. The design of the task reflected that employed by Roberts et al. (1994), which has been demonstrated to reflect variation in control of attention inhibition (Friedman \& Miyake, 2004; Miyake et al., 2000). The task measures the ability of participants to execute rapid attentional movements under conditions that require the inhibition of attention to a peripherally presented distractor stimulus, to accurately discriminate the identity of a target presented in the opposite location. The task comprised 90 trials. A schematic representation of a trial is depicted in Figure 1.

Each trial commenced with a small fixation cross presented in the centre of the screen for a duration between 1500 and $3500 \mathrm{~ms}$, in $250 \mathrm{~ms}$ increments selected at random without replacement. Next, the fixation cross was removed and simultaneously the distractor cue stimulus was presented for $225 \mathrm{~ms}$. The stimulus was a black square, $20 \mathrm{~mm} \times 20 \mathrm{~mm}$ in size, and presented $85 \mathrm{~mm}$ horizontally to the left or right of the initial fixation cross with equal frequency across trials. Following this, a visual target was presented in the screen position opposite the cue for $150 \mathrm{~ms}$. The target was a small black arrow, $5 \mathrm{~mm}$ in length, pointing left, right, or upward with equal frequency. The target arrow was masked with the icon '\#\#'. Participants were required to report the direction of the target arrow by pressing the corresponding left, right, or up arrow key on their keyboard. Following the response, the screen was cleared, and the next trial began following a 500ms inter-trial interval.

The brief exposure duration of the target is designed to ensure that successful discrimination of the cue identity requires participants to inhibit the execution of reflexive attentional movements toward the cue stimulus, to rapidly direct attention to the position of the target. Performance on the task is indexed via the number of correctly discriminated 
targets, such that a greater number of correctly discriminated targets indicates a greater level of attentional control.

The assessment task was preceded by a block of 20 practice trials. To familiarise participants with the speed of the task, practice trials presented the target arrow on screen for a gradually decreasing duration across trials $(250 \mathrm{~ms}$, to $200 \mathrm{~ms}$, to $150 \mathrm{~ms})$, and informed participants of any incorrect responses during the inter-trial interval.

\section{Procedure}

Upon accepting an online invitation to participate in the study, participants accessed the study via an online link using their personal computer. All assessments were delivered via the Inquisit Web platform. Participants were first presented information on the requirements of the study and provided informed consent. Participants next provided demographic information before completing a set of questionnaire measures that included the DASS-21. Additional questionnaires were completed for separate research projects. Participants then completed the masked-target antisaccade assessment task, followed by additional cognitive assessments completed for separate research projects. The antisaccade assessment task was always delivered prior to delivery of other tasks, and immediately preceded by detailed instructions on its requirements. Upon conclusion of the cognitive tasks, participants received debriefing information. The duration of the entire study protocol was approximately 30 minutes.

\section{Results}

\section{Participant exclusion and descriptive statistics}

A participant exclusion criterion was employed with the intention of excluding individuals that did not understand the requirements of the masked-target antisaccade task or follow the task instructions. Thus, a proportion of correct responses greater than $40 \%$ (36 
correct responses) was required for participants to be included in data analysis ${ }^{1}$. Seventyseven participants demonstrated a proportion below this threshold (range, $21 \%-40 \%$ ). Thus, subsequent statistical analysis was conducted on the remaining 265 participants. Descriptive statistics of demographic measures, DASS-Anxiety Scale scores, and the proportion of trials with correct responses on the antisaccade assessment task are present in Table 1.

\section{Internal Reliability of the Masked-target Antisaccade Assessment Task}

The internal reliability participants responses on the masked-target antisaccade assessment task was computed using a split-half multi-permutation approach, estimated across 5000 random split-halves (Parsons, 2020). The resulting Spearman-Brown corrected internal reliability estimate of the proportion of correct responses on the task was $r_{\mathrm{SB}}=.86$, $\mathrm{Cl}_{95 \%}[.84-.88]$. This clearly indicates a high level of internal reliability for the task measure. Association between Anxiety and Performance on the Masked-target Antisaccade

\section{Assessment Task}

To determine the simple association between participants' level of anxiety vulnerability and performance on the Masked-target Antisaccade Assessment Task, the correlation between DASS-Anxiety Scale scores and the proportion of trials with correct responses on the task was computed. This revealed a significant association between the two measures, $r=-.15, p=.016$, indicating that elevated levels of anxiety vulnerability were associated with a lower proportion of correction response on the Masked-target Antisaccade Assessment Task.

\footnotetext{
${ }^{1}$ Accuracy above this threshold holds less than $5 \%$ probability of occurring in the case of random responding on the task, as per a binomial distribution.
} 
To determine the manner in which participants' level of anxiety vulnerability predicted performance on the Masked-target Antisaccade Assessment Task, a logistic regression was conducted. The regression model included proportion of trials with correct responses as the dependant variable and DASS-21 Anxiety Scale scores as the predictor variable. The results of the regression analysis are present in Table 2.

The results revealed a significant effect of DASS-21 Anxiety Scale scores, such that greater DASS-21 Anxiety Scale scores predicted reduced performance on the antisaccade assessment task. Specifically, the results demonstrated that with every 1-point increase in score on the DASS-21 Anxiety Scale the odds of a correct response was reduced by $1.8 \%$. In addition, the model's prediction of the proportion of correct responses for each possible score on the DASS-21 was computed. This revealed a decline in the proportion of trials with correct responses between individuals scoring 0 (predicted proportion $=.68$ ) and individuals scoring 21 (predicted proportion $=.60$ ) on the DASS-21 Anxiety Scale. An illustration of the observed and predicted proportion of trials with correct responses across participants is presented in Figure 2.

\section{Discussion}

The aim of the present study was to determine whether the masked-target antisaccade assessment task is sensitive to anxiety-linked differences in attentional control and to assess the task's internal reliability. Results revealed greater levels of anxiety vulnerability predicted declining levels of performance on the task. This association is consistent with predictions made by contemporary theories of anxiety-linked differences in attentional processing (Eysenck \& Derakshan, 2011) and consistent with patterns of anxietylinked impairment in attentional control observed by studies using eye-tracking variants of the antisaccade task (Ansari \& Derakshan, 2010, 2011; Basanovic et al., 2018; Derakshan et 
al., 2009; Myles et al., 2020). Thus, the findings of the present study indicate that the masked-target antisaccade assessment task represents a methodology that is sensitive to anxiety-linked impairment in attentional control.

The analyses revealed that the magnitude of the simple association between performance on the masked-target antisaccade task and levels of anxiety vulnerability was small $(r=-.15)$. Previous research examining the association between anxiety vulnerability and antisaccade performance using eye-tracking measures has demonstrated larger effect sizes (Basanovic et al., 2018; Wright et al., 2014). While larger effects may be expected given these studies' use of extreme group designs, it is important for researchers to consider that the association demonstrated in the present study likely requires are large number of participants to detect reliably.

The present study also revealed the masked-target antisaccade task holds a high level of internal consistency in its measurement $\left(r_{\mathrm{SB}}=.86\right)$. Though the observed level of internal reliability is lower than some levels reported for eye-movement measures recorded during the antisaccade task $\left(r_{\mathrm{SB}}=.95\right.$, Myles et al., 2020), the present task has demonstrated much greater reliability as compared to existing approaches used to measure antisaccade performance without eye-movement recording ( $r_{\mathrm{SB}}=.59$, Basanovic et al., 2020). Researchers have noted the constraints of poor or unknown psychometric reliability of cognitive assessment measures (Parsons et al., 2019), and have increasingly called for experiment designs that overcome these limitations. The present findings indicate the masked-target antisaccade task represents a psychometrically reliable measurement option that can enable the capacity of investigators to meet these challenges when investigating anxiety-linked differences in attentional control. 
Though previous studies have demonstrated that performance on the masked-target antisaccade task is associated with other measures of cognitive control, supporting its validity as a measure of attention control (Friedman \& Miyake, 2004; Miyake et al., 2000), researchers have not compared participants' performance on the masked-target antisaccade task with their performance on eye-movement assessment variants of the antisaccade task. Thus, the degree to which measures on each task are convergent across individuals who vary in anxiety-vulnerability is unclear. Future research could determine the convergence of these measures by assessing the association between performance on each task amongst individuals who vary in anxiety vulnerability. To the extent that the measures are associated with one another and predict common variance in anxiety vulnerability, this would support the possibility that the tasks are sensitive to the same anxiety-linked difference in attention control.

For the moment however, the present findings indicate the masked-target antisaccade task provides a psychometrically reliable, low-cost, mobile, and scalable assessment of anxiety-linked differences in attentional control. It is hoped that the findings will aid researchers by affording a means to overcome existing barriers to robustly investigating anxiety-linked individual differences in attentional control, and so will facilitate research into this relationship into the future. 


\section{Open Practices Statement}

The data and materials for this study are available at https://osf.io/mc7zg/ This study was not preregistered.

\section{Declarations}

- Funding: The research reported in this manuscript was supported in part by the Australian Research Council (Grant \#: FL170100167).

- Conflicts of interest/Competing interests: The authors have no conflicts of interest to declare that are relevant to the content of this article.

- Ethics approval: Ethics approval was obtained from the human research ethics committees of The University of Western Australia and the University of Sussex.

- Consent to participate: Informed consent was obtained from all individual participants included in the study.

- Consent for publication: All participants provided informed consent for publication of the data reported in this article.

- Availability of data and materials: The datasets relating to analyses reported in the manuscript are available at: https://osf.io/mc7zg/

- Code availability: The script of the assessment task developed for the current study is available at: $\underline{\text { https://osf.io/mc7zg/ }}$

- Authors' contributions: Not applicable. 


\section{References}

Ansari, T. L., \& Derakshan, N. (2010). Anxiety impairs inhibitory control but not volitional action control. Cognition \& Emotion, 24(2), 241-254. https://doi.org/10.1080/02699930903381531

Ansari, T. L., \& Derakshan, N. (2011). The neural correlates of impaired inhibitory control in anxiety. Neuropsychologia, 49(5), 1146-1153. https://doi.org/10.1016/j.neuropsychologia.2011.01.019

Ansari, T. L., Derakshan, N., \& Richards, A. (2008). Effects of anxiety on task switching: Evidence from the mixed antisaccade task. Cognitive, Affective, \& Behavioral Neuroscience, 8(3), 229-238. https://doi.org/10.3758/CABN.8.3.229

Antony, M. M., Bieling, P. J., Cox, B. J., Enns, M. W., \& Swinson, R. P. (1998). Psychometric properties of the 42-item and 21-item versions of the Depression Anxiety Stress Scales in clinical groups and a community sample. Psychological Assessment, 10(2), 176-181. https://doi.org/10.1037/1040-3590.10.2.176

Bardeen, J. R., \& Orcutt, H. K. (2011). Attentional control as a moderator of the relationship between posttraumatic stress symptoms and attentional threat bias. Journal of Anxiety Disorders, 25(8), 1008-1018. https://doi.org/10.1016/j.janxdis.2011.06.009

Basanovic, J., Kaiko, I., \& MacLeod, C. (2020). Change in Attentional Control Predicts Change in Attentional Bias to Negative Information in Response to Elevated State Anxiety. Cognitive Therapy and Research, 1990. https://doi.org/10.1007/s10608-020-10176-3

Basanovic, J., Notebaert, L., Clarke, P. J. F., MacLeod, C., Jawinski, P., \& Chen, N. T. M. (2018). Inhibitory attentional control in anxiety: Manipulating cognitive load in an antisaccade task. PLOS ONE, 13(10), e0205720. https://doi.org/10.1371/journal.pone.0205720

Basanovic, J., Notebaert, L., Grafton, B., Hirsch, C. R., \& Clarke, P. J. F. (2017). Attentional control predicts change in bias in response to attentional bias modification. Behaviour Research and Therapy, 99, 47-56. https://doi.org/10.1016/j.brat.2017.09.002

Bates, D., Mächler, M., Bolker, B., \& Walker, S. (2015). Fitting Linear Mixed-Effects Models Using Ime4. Journal of Statistical Software, 67(1), 883-885. https://doi.org/10.18637/jss.v067.i01

Chen, N. T. M., Clarke, P. J. F., Watson, T. L., MacLeod, C., \& Guastella, A. J. (2014). Biased saccadic responses to emotional stimuli in anxiety: An antisaccade study. PLOS ONE, 9(2). https://doi.org/10.1371/journal.pone.0086474

Derakshan, N., Ansari, T. L., Hansard, M., Shoker, L., \& Eysenck, M. W. (2009). Anxiety, inhibition, efficiency, and effectiveness: An investigation using the Antisaccade task. Experimental Psychology, 56(1), 48-55. https://doi.org/10.1027/1618-3169.56.1.48

Derakshan, N., \& Eysenck, M. W. (2009). Anxiety, processing efficiency, and cognitive performance: New developments from attentional control theory. European Psychologist, 14(2), 168-176. https://doi.org/10.1027/1016-9040.14.2.168

Derryberry, D., \& Reed, M. A. (2002). Anxiety-related attentional biases and their regulation by attentional control. Journal of Abnormal Psychology, 111(2), 225-236. https://doi.org/10.1037/0021-843X.111.2.225

Ettinger, U., Kumari, V., Crawford, T. J., Davis, R. E., Sharma, T., \& Corr, P. J. (2003). Reliability of smooth pursuit, fixation, and saccadic eye movements. Psychophysiology, 40(4), 620-628. https://doi.org/10.1111/1469-8986.00063

Eysenck, M. W., \& Derakshan, N. (2011). New perspectives in attentional control theory. Personality and Individual Differences, 50(7), 955-960. 
https://doi.org/10.1016/j.paid.2010.08.019

Eysenck, M. W., Derakshan, N., Santos, R., \& Calvo, M. G. (2007). Anxiety and cognitive performance: Attentional control theory. Emotion, 7(2), 336-353. https://doi.org/10.1037/1528-3542.7.2.336

Fajkowska, M., \& Derryberry, D. (2010). Psychometric properties of Attentional Control Scale: The preliminary study on a Polish sample. Polish Psychological Bulletin, 41(1), 17. https://doi.org/10.2478/s10059-010-0001-7

Friedman, N. P., \& Miyake, A. (2004). The Relations Among Inhibition and Interference Control Functions: A Latent-Variable Analysis. Journal of Experimental Psychology: General, 133(1), 101-135. https://doi.org/10.1037/0096-3445.133.1.101

Guitton, D., Buchtel, H. A., \& Douglas, R. M. (1985). Frontal lobe lesions in man cause difficulties in suppressing reflexive glances and in generating goal-directed saccades. Experimental Brain Research, 58(3), 455-472. https://doi.org/10.1007/BF00235863

Hallett, P. E. (1978). Primary and secondary saccades to goals defined by instructions. Vision Research, 18(10), 1279-1296. https://doi.org/10.1016/0042-6989(78)90218-3

Kane, M. J., Bleckley, M. K., Conway, A. R. A., \& Engle, R. W. (2001). A controlled-attention view of working-memory capacity. Journal of Experimental Psychology: General, 130(2), 169-183. https://doi.org/10.1037//0096-3445.130.2.169

Lovibind, S. H., \& Lovibond, P. F. (1995). Manual for the Depression Anxiety \& Stress Scales (2nd ed.). Psychology Foundation.

Miyake, A., Friedman, N. P., Emerson, M. J., Witzki, A. H., Howerter, A., \& Wager, T. D. (2000). The Unity and Diversity of Executive Functions and Their Contributions to Complex "Frontal Lobe" Tasks: A Latent Variable Analysis. Cognitive Psychology, 41(1), 49-100. https://doi.org/10.1006/cogp.1999.0734

Muris, P., de Jong, P. J., \& Engelen, S. (2004). Relationships between neuroticism, attentional control, and anxiety disorders symptoms in non-clinical children. Personality and Individual Differences, 37(4), 789-797. https://doi.org/10.1016/j.paid.2003.10.007

Myles, O., Grafton, B., \& MacLeod, C. (2020). Anxiety \& inhibition: dissociating the involvement of state and trait anxiety in inhibitory control deficits observed on the antisaccade task. Cognition and Emotion, 34(8), 1746-1752.

https://doi.org/10.1080/02699931.2020.1802229

Ólafsson, R. P., Smári, J., Guđmundsdóttir, F., Ólafsdóttir, G., Harđardóttir, H. L., \& Einarsson, S. M. (2011). Self reported attentional control with the Attentional Control Scale: Factor structure and relationship with symptoms of anxiety and depression. Journal of Anxiety Disorders, 25(6), 777-782. https://doi.org/10.1016/j.janxdis.2011.03.013

Parsons, S. (2020). Splithalf: Robust Estimates of Split Half Reliability (0.71). https://doi.org/10.6084/m9.figshare.11956746.v4

Parsons, S., Kruijt, A.-W., \& Fox, E. (2019). Psychological Science Needs a Standard Practice of Reporting the Reliability of Cognitive-Behavioral Measurements. Advances in Methods and Practices in Psychological Science, 2(4), 378-395. https://doi.org/10.1177/2515245919879695

Quigley, L., Wright, C. A., Dobson, K. S., \& Sears, C. R. (2017). Measuring Attentional Control Ability or Beliefs? Evaluation of the Factor Structure and Convergent Validity of the Attentional Control Scale. Journal of Psychopathology and Behavioral Assessment, 39(4), 742-754. https://doi.org/10.1007/s10862-017-9617-7

Reinholdt-Dunne, M. L., Mogg, K., Benson, V., Bradley, B. P., Hardin, M. G., Liversedge, S. P., Pine, D. S., \& Ernst, M. (2012). Anxiety and selective attention to angry faces: An 
antisaccade study. Journal of Cognitive Psychology, 24(1), 54-65. https://doi.org/10.1080/20445911.2011.560111

Reinholdt-Dunne, M. L., Mogg, K., \& Bradley, B. P. (2009). Effects of anxiety and attention control on processing pictorial and linguistic emotional information. Behaviour Research and Therapy, 47(5), 410-417. https://doi.org/10.1016/j.brat.2009.01.012

Reinholdt-Dunne, M. L., Mogg, K., \& Bradley, B. P. (2013). Attention control: Relationships between self-report and behavioural measures, and symptoms of anxiety and depression. Cognition \& Emotion, 27(3), 430-440. https://doi.org/10.1080/02699931.2012.715081

Roberts, R. J., Hager, L. D., \& Heron, C. (1994). Prefrontal cognitive processes: Working memory and inhibition in the antisaccade task. Journal of Experimental Psychology, 123(4), 374-393. https://doi.org/10.1037/0096-3445.123.4.374

Schoorl, M., Putman, P., Van Der Werff, S., \& Van Der Does, A. J. W. (2014). Attentional bias and attentional control in Posttraumatic Stress Disorder. Journal of Anxiety Disorders, 28(2), 203-210. https://doi.org/10.1016/j.janxdis.2013.10.001

Shi, R., Sharpe, L., \& Abbott, M. J. (2019). A meta-analysis of the relationship between anxiety and attentional control. Clinical Psychology Review, 101754. https://doi.org/10.1016/J.CPR.2019.101754

Todd, J., Notebaert, L., \& Clarke, P. J. F. (2020). Does the attentional control scale measure attentional control? Evidence of no relationship with antisaccade performance. PsychArchives [PrePrint], 1-19. https://doi.org/http://dx.doi.org/10.23668/psycharchives.3116

Unsworth, N., Spillers, G. J., Brewer, G. A., \& McMillan, B. (2011). Attention control and the antisaccade task: A response time distribution analysis. Acta Psychologica, 137(1), 90100. https://doi.org/10.1016/j.actpsy.2011.03.004

Wieser, M. J., Pauli, P., \& Mühlberger, A. (2009). Probing the attentional control theory in social anxiety: An emotional saccade task. Cognitive, Affective, \& Behavioral Neuroscience, 9(3), 314-322. https://doi.org/10.3758/CABN.9.3.314

Williams, P. G., Rau, H. K., Suchy, Y., Thorgusen, S. R., \& Smith, T. W. (2017). On the validity of self-report assessment of cognitive abilities: Attentional control scale associations with cognitive performance, emotional adjustment, and personality. Psychological Assessment, 29(5), 519-530. https://doi.org/10.1037/pas0000361

Wright, C. A., Dobson, K. S., \& Sears, C. R. (2014). Does a high working memory capacity attenuate the negative impact of trait anxiety on attentional control? Evidence from the antisaccade task. Journal of Cognitive Psychology, 26(4), 400-412.

https://doi.org/10.1080/20445911.2014.901331 


\section{Figure 1}

Schematic of the progression of a single trial presented in the Masked-target Antisaccade Performance Assessment Task. Note figure not to scale.

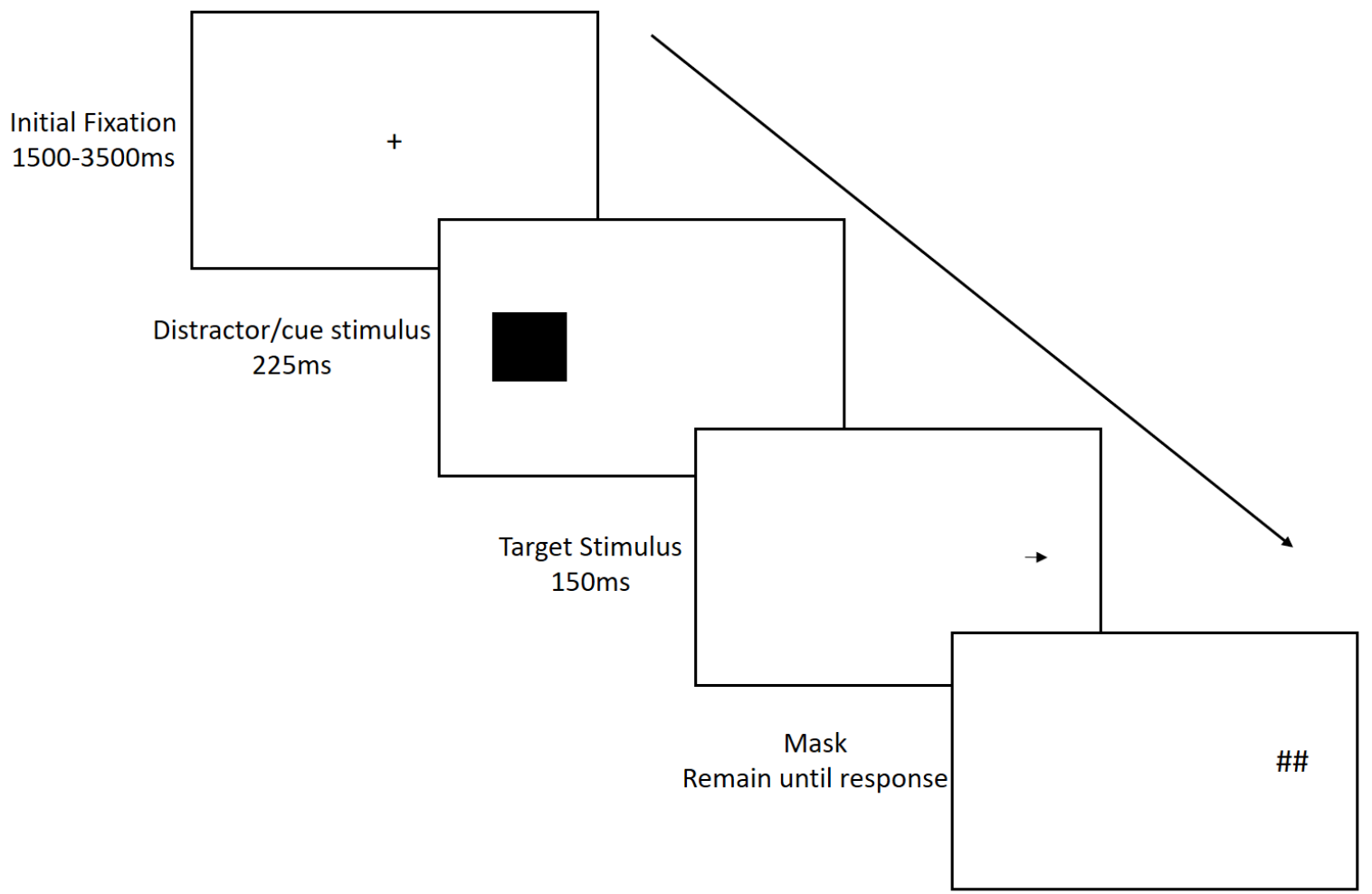


Table 1

Descriptive statistics of measures of demographic measures and measures of the proportion of correct responses on the antisaccade assessment task. $\mathrm{N}=265$.

\begin{tabular}{lccc}
\hline Measure & Female & Male & Non-Binary \\
\hline Gender & 184 & 78 & 3 \\
\cline { 2 - 3 } & Mean (SD) & Range \\
\cline { 2 - 3 } Age (years) & $20.79(4.99)$ & $17-58$ \\
DASS-21 - Anxiety Subscale & $5.40(4.76)$ & $0-20$ \\
Antisaccade Assessment Task & $.66(.13)$ & $.41-.97$ \\
(proportion of responses correct) & & \\
\hline
\end{tabular}


Table 2

Results of the logistic regression model predicting number of correct responses in the masked-target antisaccade assessment task.

\section{Response Correct}

\begin{tabular}{lccc} 
Predictors & Odds Ratio & $\mathrm{Cl}(95 \%)$ & $p$ \\
\hline (Intercept) & 2.144 & $2.059-2.234$ & $<.001^{*}$ \\
DASS-21 Anxiety Scale Score & 0.982 & $0.977-0.988$ & $<.001^{*}$ \\
$\mathrm{~N}=265$ & & & \\
\hline
\end{tabular}




\section{Figure 2}

Illustration of the association between participant's antisaccade performance, as indexed by the proportion of responses correct, and DASS-21 anxiety scale scores. Line represents values predicted by the computed regression model, with shaded band representing $95 \%$ confidence interval. Points represent individual participants, with darker shades representing overlap.

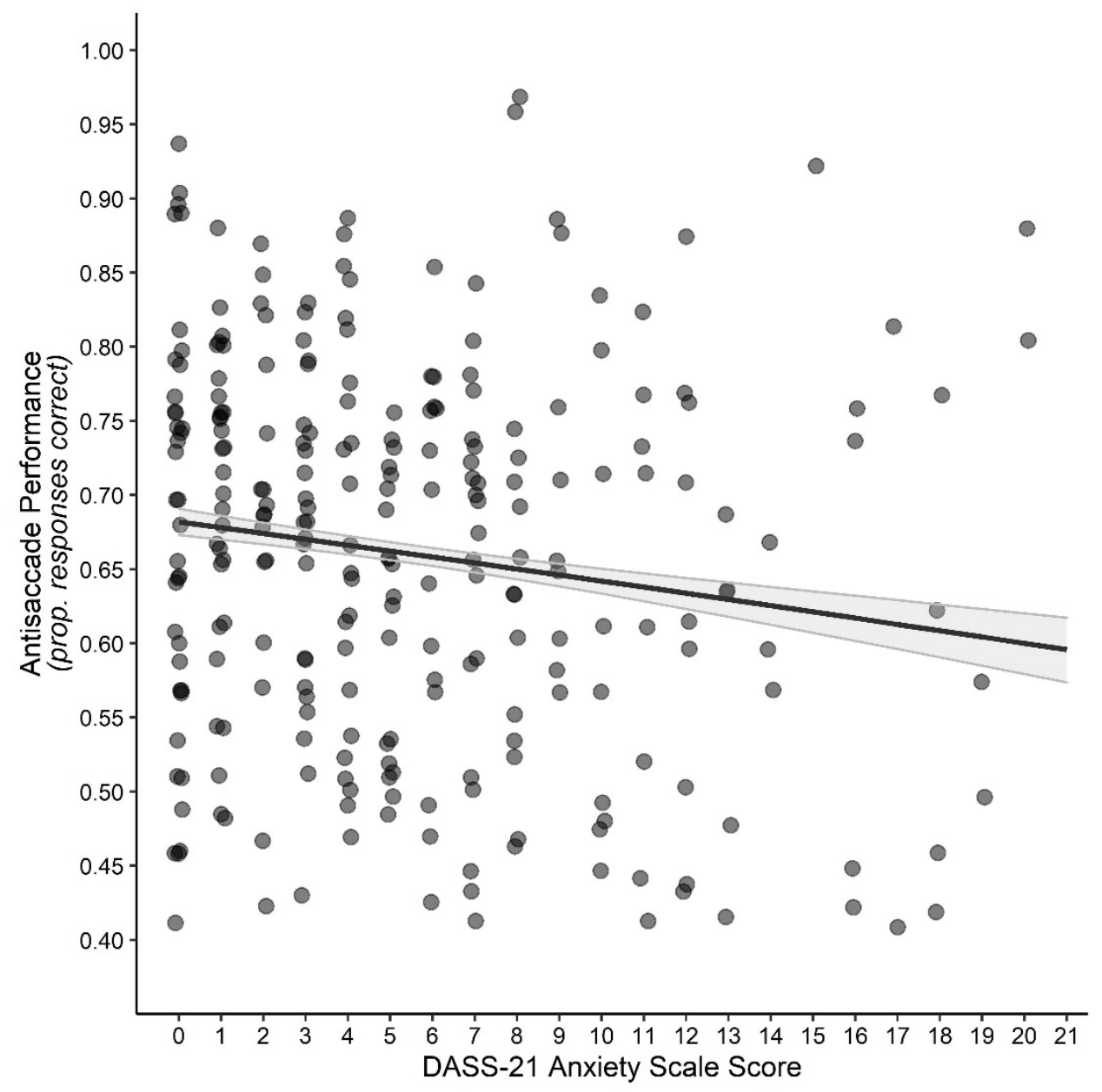




\section{Open Practices Statement}

The data and materials for this study are available at https://osf.io/mc7zg/

This study was not preregistered.

\section{Declarations}

- Funding: The research reported in this manuscript was supported in part by the Australian Research Council (Grant \#: FL170100167).

- Conflicts of interest/Competing interests: The authors have no conflicts of interest to declare that are relevant to the content of this article.

- Ethics approval: Ethics approval was obtained from the human research ethics committees of The University of Western Australia and the University of Sussex.

- Consent to participate: Informed consent was obtained from all individual participants included in the study.

- Consent for publication: All participants provided informed consent for publication of the data reported in this article.

- Availability of data and materials: The datasets relating to analyses reported in the manuscript are available at: https://osf.io/mc7zg/

- Code availability: The script of the assessment task developed for the current study is available at: $\underline{\text { https://osf.io/mc7zg/ }}$

- Authors' contributions: Not applicable. 